\title{
Erratum to: Pseudarthrose du scaphoïde
}

\section{Erratum to: Scaphoid Nonunion}

\author{
D. Tourdias \\ (C) SFMU et Lavoisier SAS 2016
}

Erratum to : Ann. Fr. Med. Urgence DOI 10.1007/s13341-016-0645-9

La légende de la Figure 1 est erronnée ; il fallait lire :

Fig. 1 : Pseudarthrose du scaphoïde de découverte fortuite sur des clichés standard de poignet. A. cliché de face : notez l'aspect typique (flèche) avec écart interfragmentaire important, présence d'une géode sur la berge proximale et d'une ostéocondensation de la berge distale ; B. cliché de profil : il existe une bascule postérieure pathologique du lunatum témoignant d'une DISI avec des angles scapholunaire $(\alpha)$ et radiolunaire $(\beta)$ augmentés (respectivement mesurés à $84^{\circ}$ et $24^{\circ}$ ). L'axe du lunatum (ligne blanche) est perpendiculaire à la tangente qui relie ses deux cornes antérieure et postérieure (ligne blanche pointillée). L'axe du scaphoïde (ligne noire pleine), souvent difficile à tracer, correspond à la tangente au tubercule du scaphoïde et à son pôle proximal ; il permet la mesure de l'angle scapholunaire qui est habituellement comprise entre $30^{\circ}$ et $60^{\circ}$. L'axe du radius (ligne noire pointillée) est toujours bien individualisable ; il permet de mesurer l'angle radiolunaire qui est normalement compris entre $10^{\circ}$ et $15^{\circ}$ 